\title{
Immer Ärger mit der Materialität? - Politische Ökologie und das Dispositiv der Dürre im Nordosten Brasiliens
}

\section{Tobias Schmitt}

\author{
Institut für Geographie, Universität Hamburg, Bundesstr. 55, 20146 Hamburg, Germany
}

Correspondence to: Tobias Schmitt (tobias.schmitt@uni-hamburg.de)

Received: 8 March 2016 - Revised: 17 August 2016 - Accepted: 23 August 2016 - Published: 17 October 2016

Kurzfassung. While the role of materiality was understudied in most social sciences, there was a sensibility for these issues in political ecology. The different approaches of political ecology focused on the political character of ecology and the ecological (and thus material) character of politics. But a conceptual framework that captures the different dimensions of the societal nature relations was seldom explicitly elaborated. Following the considerations of Michel Foucault, this article explores whether dispositive analysis as a concept and as a method offers a way to integrate both social and material conditions into studies of political ecology. By examining water infrastructure and the dispositive of drought in Northeastern Brazil, this paper displays how dispositive analysis is a means to identify different elements, their autonomies as well as their interconnectedness. Focusing on the entanglements of discourses, institutionalizations, subjectivity, practices and materiality allows capturing the materiality of discourses and the discursivity of material orders.

\section{Einleitung}

Mit den unter dem Schlagwort „,cultural turn“ zusammengefassten Verschiebungen in den Sozialwissenschaften und der vermehrten Fokussierung auf Sprache, Zeichen, Symbole und Bilder als zentrale Elemente zum Verständnis von Welt, wurde die materiell-stoffliche Dimension zunehmend vernachlässigt oder gar ganz aus den theoretischen Überlegungen und vorgenommenen Analysen ausgeblendet (Kazig und Weichhart, 2009:109; Pels et al., 2002:5; Namberger, 2013:135). Auch in der Humangeographie bestand die Gefahr, die materielle Welt und ihre Bedeutung für (kulturelle) Praktiken aus dem Blickfeld zu verlieren (Kazig und Weichhart, 2009:110). Gegenstände, Körper oder Landschaften wurden dabei am ehesten noch als passives, unbelebtes Rohmaterial thematisiert, in das sich Diskurse und Machtverhältnisse einschreiben, und somit lediglich als Resultate sozialer Prozesse begriffen (Lykke, 2013:44; Pels et al., 2002:5). Als Reaktion darauf wurde eine Re-Materialisierung der Forschung eingefordert, um die vielfältigen Verwobenheiten zwischen materiellen und sozialen Prozessen (wieder) benennen zu können (Bakker und Bridge, 2006:5; Mattisek und Wiertz, 2014:157; Kazig und Weichhart, 2009:110): „After poststructuralism and constructivism had melted everything that was solid into air, it was perhaps time that we noticed once again the sensuous immediacy of the objects we live, work and converse with, $[\ldots]$ which bind us as much as we bind them" (Pels et al., 2002:1).

Durch eine Rückbesinnung auf das Materielle als konstitutives Element sozialer Wirklichkeit bei einer expliziten Berücksichtigung der gegenseitigen Bedingungsverhältnisse zwischen Sozialem und Materiellem besteht jedoch die Möglichkeit, Gegenstände, Körper, gebaute Umwelt usw. in die Analysen zu integrieren, ohne erneut in Essentialisierungen und Dichotomisierungen zu verfallen. Indem dabei die Eigenständigkeit von sozialen und materiellen Prozessen betont und gleichzeitig auf ihre unausweichliche gegenseitige Durchdringung bestanden wird, kann über eine additive Aufzählung verschiedener Dimensionen der Wirklichkeitskonstitution hinaus gegangen werden.

Gerade in der politischen Ökologie, die aus einer Kritik an einer einseitigen und unpolitischen Bearbeitung von sogenannten Umweltproblemen entstanden ist, besteht eine lange Tradition, die gegenseitigen Wechselverhältnisse zwischen ökologischen und sozialen Prozessen aus ganz unterschiedlichen erkenntnistheoretischen Positionen in den Blick zu neh- 
men (Robbins, 2004:7 f.). Eine der Stärken der politischen Ökologie besteht dabei unzweifelhaft darin, konzeptionelle Ansätze mit konkreten empirischen Studien zu verknüpfen. Nachdem zunächst eher marxistische Ansätze innerhalb der politischen Ökologie überwogen, fanden seit den 1980er Jahren verschiedene, mit dem Präfix ,post“ markierte Ansätze (poststrukturalistische, postmarxistische, postkoloniale) Einzug in die Debatte (Escobar, 2010:91). Somit rückten diskursanalytische Methoden und Fragen nach der Produktion von Wissen und die Konstitution von Bedeutung über Natur und Materialität mehr und mehr in den Vordergrund. Weniger Beachtung fand bisher jedoch das Konzept einer Dispositivanalyse, das vor allem auf Überlegungen von Michel Foucault zurück zu führen ist (Foucault, 1978). Hierbei werden nicht sprachliche Äußerungsmodalitäten auf der einen und materielle Ordnungen auf der anderen Seite untersucht. Vielmehr geht es um die machtstrategischen Verbindungen zwischen Diskursen, Institutionalisierungen, Subjektpositionen, Praktiken und Materialisierungen (Bührmann und Schneider, 2008). Im Fokus stehen folglich Fragen nach dem gegenseitigen Hervorbringen von diskursiven und materiellen Formationen und nach den Auswirkungen ihres Zusammenwirkens.

Ziel dieses Beitrags ist es, das Konzept des Dispositivs und die Methodik der Dispositivanalyse als Zugang zu erarbeiten und damit das Verhältnis zwischen Gesellschaft und Natur analytisch zu fassen. Als empirisches Beispiel dienen dabei die Herstellung von und der Umgang mit Dürreverhältnissen im Nordosten Brasiliens. Insbesondere wird dabei auf das Verhältnis zwischen Materialität und Diskursivität am Beispiel der Wasserinfrastruktureinrichtungen - Staudämme, Kanäle, Pumpen etc. - eingegangen. An ihnen kann aufgezeigt werden, wie materielle Ensembles und Diskurse sowie institutionelle Arrangements sich gegenseitig hervorbringen und zusammen diskursive und nicht-diskursive Praktiken strukturieren. So wird in ihnen sowohl die Materialität des Diskurses als auch die Diskursivität des Materiellen deutlich. Über ein solches Dispositiv werden bestimmte Prozesse und Entwicklungen erzeugt, die jedoch nicht auf einzelne Elemente sondern nur auf ihr Zusammenspiel zurückzuführen sind. Darüber hinaus kann ein Verständnis der Eigenständigkeit von Natur und der Bruch mit einer rein konstruktivistischen Sichtweise herausgearbeitet werden. Gerade dann, wenn Naturaneignung scheitert, wenn eine einfache Übertragung von Diskursen und Ideen auf materielle Gegebenheiten ungeahnte Ergebnisse zeitigt, wenn Stauseen austrocknen oder Wasser verschmutzt wird, wird der eigenständige Charakter von Materialität sichtbar.

Zunächst soll jedoch in einer kurzen Skizze aufgezeigt werden, wie Materialität innerhalb der politischen Ökologie - insbesondere auch in Bezug auf Wasser und Wasserinfrastruktur - verhandelt wird, und welche Herausforderungen sich daraus für eine konzeptionelle Weiterentwicklung ergeben. Dabei wird jedoch vor allem auf Ansätze aus dem angloamerikanischen Raum verwiesen, sodass Debatten insbesondere aus den Ländern des Globalen Südens und außerakade- mische Diskussionen unbenannt bleiben. Ihre Einbeziehung stellt eine Herausforderung für zukünftige Auseinandersetzungen und Forschungsprojekte dar.

\section{2 „Matter matters“ innerhalb der politischen Ökologie}

Gemeinsamer Ausgangspunkt der frühen Arbeiten, die sich auf den Begriff der politischen Ökologie beziehen, war die Kritik an der ontologischen Trennung zwischen Materialität (Natur) und Gesellschaft (Kultur) seit der europäischen Aufklärung (Castree, 2001:6). Gleichzeitig grenzte man sich von einem naturdeterministischen und (neo)malthusianischen Verständnis natürlicher Grenzen und der Vorstellung von Materialität als ,Ausdruck bestimmter Zwangsgesetzlichkeiten“ (Wissen, 2011:108) ab. Demgegenüber gehen die Ansätze der politischen Ökologie von der Annahme aus, dass Natur keine externe, objektive Einheit, sondern ein Produkt sozialer und folglich auch politischer Prozesse darstellt (Budds, 2004:32). Ökologische Prozesse, wie etwa Bodenerosion und Desertifikation, werden dabei als inhärenter Bestandteil von polit-ökonomischen und historischen Prozessen verstanden (Blaikie und Brookfield, 1987).

Viele Arbeiten der ersten Generation der politischen Ökologie der 1970er und 1980er Jahre sind von einer historischmaterialistischen Tradition geprägt (Köhler, 2008:852). Dabei bilden der Begriff des Stoffwechsels zwischen Mensch und Natur als ,ewige[n] Naturbedingung des menschlichen Lebens" (Marx, 1968:198) und der Begriff von Arbeit als Prozess der Aneignung und Transformation von Natur zentrale Kategorien zur Analyse und Beschreibung des MenschNatur-Verhältnisses. Aus ökomarxistischer Perspektive stellt die Materialität von Natur daher einen der grundlegenden Widersprüche der kapitalistischen Produktionsweise dar. Da die Verwertungslogik des Kapitals mit der Reproduktionslogik von Natur nicht übereinstimmt ,produziert der Kapitalismus Natur negativ, das heißt in Gestalt von Umweltzerstörung; in letzter Konsequenz produziert er sie als seine eigene Schranke“" (Wissen, 2011:117).

Wichtig ist hierbei der Begriff der (kapitalistischen) Produktion von Natur, den vor allem Smith (1984) stark gemacht hat. Dabei wird Natur in Anlehnung an Marx als stofflichmaterielles und zugleich historisch-gesellschaftliches Produkt verstanden, das über spezifische Praktiken hergestellt wird. Jedoch werden durch den Fokus auf die Prozesse der sozialen Produktion von Natur die Eigengesetzlichkeiten materieller Bedingungen vernachlässigt: ,The production of nature thesis tries to solve this historically by ,internalizing nature' as a social product but [...] ends up squeezing out any productive or generative role for ecology or biophysical processes“" (Bakker und Bridge, 2006:9).

Demgegenüber macht Harvey (2007) ein dialektisches Verständnis der Mensch-Natur-Beziehungen stark. In Anlehnung an das von Alfred North Whitehead formulierte 
Prinzip des ,being is constituted by becoming“ (Whitehead in Harvey, 2007:54) betont er die gegenseitige Konstituierung von Subjekt und Objekt, von Sozialem und Materiellem, wobei weniger die Analyse von Dingen, (Öko)Systemen oder Strukturen, sondern vielmehr von Prozessen, Strömungen und Beziehungen im Vordergrund stehen (Harvey, 2007:49 f.).

Auch Castree (2001) geht von einer unausweichlichen Verwobenheit zwischen Sozialem und Materiellem aus. Mit seinem Konzept der sozialen Natur (social nature) greift er Smith's These der Produktion von Natur auf, besteht aber mit Bezugnahme auf Harvey - auf die unvermeidbare und unkontrollierbare Eigenständigkeit von Materialität: ,,nature may indeed be ,produced ' but produced nature, in turn, cannot be exploited indefinitely: it has a materiality which cannot be ignored" (Castree, 2000:29).

Wichtige Impulse für die konzeptionelle Weiterentwicklung des Mensch-Natur-Verhältnisses kamen u.a. von der Actor-Network-Theory (ANT) (Haraway, 1991; Latour, 1998). Dabei wird nicht danach gefragt, wie Gesellschaft Natur produziert, sondern wie Menschen und Dinge in Netzwerke eingebunden werden, wodurch neue hybride Formationen entstehen. Eine der bedeutendsten Neuerungen der ANT stellt die Sichtweise dar, Natur, Gegenstände, Instrumente etc. nicht als passive Einheiten zu begreifen, über die gesellschaftliche Verhältnisse vermittelt werden. Vielmehr wird ihnen über die Einbindung in Netzwerke Handlungsmacht (agency) zugeschrieben, da sie die Fähigkeit besitzen, die Verbindungen innerhalb des Netzwerkes zu stabilisieren oder zu untergraben (Murdoch, 1997:740). Diese NaturKultur-Hybride - Haraway (1991) prägte den Begriff des Cyborgs, Latour (1998) den der Quasi-Objekte - sind jedoch keine einfachen Mischwesen, sondern emergente Resultate der Netzwerkbildung, denen ein eigener ontologischer Status zugwiesen wird, ,one that ,does not resemble either that of the forlorn and passive things-in-themselves, or that of humans-among-themselves. Too social to look like the former, they remain too nonhuman to resemble the latter" (Latour in Murdoch, 1997:744).

Die Einforderung ontologischer Gleichbehandlung von Materiellem und Symbolischem und die emergente Herausbildung neuer Eigenschaften und Fähigkeiten durch die Verknüpfung von Subjekten, Institutionen, Dingen usw. in sogenannten Gefügen stellen auch für die Assemblage-Theorie in Anlehnung an die Arbeiten von Gilles Deleuze und Felix Guattari zentrale Eckpunkte dar (Mattissek und Wiertz, 2014:160). Da hier jedoch in Abgrenzung zur ANT der Sprache eine besondere Bedeutung zukommt und der Ansatz somit für diskurs- und machttheoretische Überlegungen anschlussfähig wird, sehen Annika Mattisek und Thilo Wiertz darin eine vielversprechenden Chance für die Humangeographie, den Zusammenhang zwischen symbolischen und materiellen Aspekten gesellschaftlicher Wirklichkeit konzeptionell weiter zu denken (ebd.:162).
Gemeinsam ist den unterschiedlichen Ansätzen der politischen Ökologie in erster Linie die Zurückweisung essentialistischer Vorstellungen der „Natur der Dinge“. Natur wird dabei sowohl als stofflich-materiell als auch als sozialsymbolisch begriffen. Ob der Blick jedoch vornehmlich auf die soziale Konstruktion von Natur, die gegenseitige Konstituierung von materiellen und sozialen Prozessen oder auf die Handlungsweisen sozialökologischer Hybride gelegt wird, hat sowohl Auswirkungen auf den Untersuchungsgegenstand als auch auf Forschungsmethoden und mögliche Erkenntnisgewinne. Zentrale Frage bleibt dabei, inwiefern die Eigenständigkeit von Natur ernst genommen wird, ohne in eine erneute Essentialisierung - etwa von physischen und chemischen Prozessen - zu verfallen.

Im deutschsprachigen Raum hat sich mit dem Konzept der gesellschaftlichen Naturverhältnisse, das sich in erster Linie auf die Kritische Theorie Frankfurter Schule bezieht, ein weiterer Strang innerhalb der politischen Ökologie entwickelt. Insbesondere mit dem Begriff der Nicht-Identität von Natur (Görg, 2003b) wird eine konzeptionelle Rahmung vorgeschlagen, die die Unmöglichkeit eines direkten, unvermittelten Zugangs zu Natur anerkennt und gleichzeitig der Natur eine gewisse Autonomie einräumt.

\subsection{Gesellschaftliche Naturverhältnisse}

„Der gesellschaftliche Prozess ist weder bloß Gesellschaft noch bloß Natur, sondern Stoffwechsel der Menschen mit dieser, die permanente Vermittlung beider Momente" (Adorno, 2003:221).

In diesem Zitat von Theodor W. Adorno werden bereits die Grundannahmen des Konzeptes der gesellschaftlichen Naturverhältnisse deutlich: Gesellschaft und Natur stehen in einem permanenten Vermittlungsverhältnis, sodass sie nicht ,an sich“ existieren und unabhängig voneinander erkannt werden können. Jedoch führt ein solches Vermittlungsverhältnis nicht zu einer großen Einheit, bei der beide Elemente vollständig ineinander aufgehen. Vielmehr bleiben sie in einem Spannungsverhältnis von Relation und Differenz bestehen und können als ,,aufeinander bezogene gegensätzliche Pole einer Differenz“" (Jahn und Wehling, 1998:83) begriffen werden. Als gesellschaftliche Naturverhältnisse werden ,relativ dauerhafte Beziehungsmuster“ (Becker et al., 2011:89) bezeichnet, die sowohl eine stofflich-materielle als auch eine kulturell-symbolische Dimension aufweisen, wobei eine solche Unterscheidung lediglich analytisch vorgenommen werden kann. Faktisch kann die gegenseitige Durchdringung nicht voneinander getrennt werden (Jahn und Wehling, 1998:84).

Dabei sind die gesellschaftlichen Naturverhältnisse sowohl in kulturelle Deutungszusammenhänge und Wahrnehmungsmuster eingebettet - wie sie etwa in religiösen und ästhetischen Naturbildern oder wissenschaftlichen Erklärungen zum Ausdruck kommen - als auch in bio-physische Prozesse und deren Wirkungszusammenhänge. Damit Natur 
- oder ein bestimmter Ausschnitt von Natur - eine gesellschaftliche Bedeutung erhält, braucht es immer die Vermittlung über bestimmte Repräsentationen (Wissen, 2011:121; Becker et al., 2011:78). Gleichzeitig können gesellschaftliche Naturverhältnisse nicht abgekoppelt von der Materialität und den stofflich-energetischen Eigenschaften gedacht und hergestellt werden. Die materiellen Bedingungen und Gesetzmäßigkeiten weisen gegenüber sozialen Prozessen bestimmte Widerständigkeiten auf und entziehen sich dadurch einer vollständigen Verfügbarkeit und Kontrolle (Wissen, 2011:122). Natur kann dabei somit nicht als bloße Trägerin von Bedeutung verstanden werden, sondern ist immer auch Ko-Produzentin der gesellschaftlichen Naturverhältnisse.

Die Unmöglichkeit einer rein sozialen Konstruktion von Natur und eines einseitigen Einschreibens von gesellschaftlichen Verhältnissen in die materiellen Bedingungen verweist auf die Eigenständigkeit von Natur. Mit dem Begriff der Nicht-Identität von Natur bezeichnet Görg (2003a) in Anlehnung an Theodor W. Adorno etwas, das nicht benannt werden kann, das über die identitäre Zuschreibung von Natur hinausgeht. Die Nicht-Identität ist quasi die Lücke zwischen der Benennung der Dinge und den Dingen selbst. Somit ist die Nicht-Identität nicht als eine positiv bestimmbare Objektivität oder als ontologischer Kern von Natur zu verstehen, ,,der sich bestimmen ließe, wenn man den gesellschaftlichen Einfluss gleichsam ,abzieht “" (Wissen, 2011:122). Vielmehr wird von einer Eigengesetzlichkeit von Natur ausgegangen, ohne dass diese positiv und a priori bestimmt werden kann. Diese erschließt sich erst dann, wenn gewisse Formen der Naturaneignung mit der Eigenlogik von Natur in Widerspruch geraten, wenn also die Benennung und Aneignung von Natur scheitern (Görg, 2005:234).

Demzufolge liegt der Fokus des Konzeptes auf den Wechselbeziehungen zwischen gesellschaftlichen Verhältnissen und Naturverhältnissen. Im Gegensatz etwa zur ANT wird jedoch explizit danach gefragt, wie Macht- und Herrschaftsverhältnisse an Naturverhältnisse gekoppelt sind und über diese produziert und reproduziert werden. Macht wird dabei nicht allein als ,ability of an actor to control their own interaction with the environment and the interaction of the other actors with the environment" (Bryant und Bailey, 1997:37) verstanden. Vielmehr kommen Machtverhältnisse in den vielfältigen Beziehungen zwischen sozialen und materiellen Verhältnissen zum Tragen. Insofern wird danach gefragt, wie sich Macht- und Herrschaftsverhältnisse manifestieren, in die Natur einschreiben und darüber verstetigt werden. Gleichzeitig geht es um die Prozesse und Mechanismen, über die bestimmte materielle Bedingungen Diskurse und gesellschaftliche Praktiken vorstrukturieren und folglich zu einem Medium sozialer Herrschaft werden (Görg, 2003b:201; Köhler und Wissen, 2010:223). Darüber hinaus wird in Anlehnung an die Kritische Theorie das Verhältnis zwischen Natur, Gesellschaft und Subjekt ausgelotet. Insbesondere Theodor W. Adorno und Max Horkheimer haben auf das enge Verhältnis zwischen der Herrschaft des Menschen über die Natur, über andere Menschen und der Verinnerlichung von Herrschaftsverhältnissen im Subjekt hingewiesen (Adorno, 1950:125; Horkheimer, 1967:94).

\subsection{Wasser als emblematische Materialität}

Ganz besonders prominent wird das wechselseitige Verhältnis zwischen materieller Natur und nicht-materiellen gesellschaftlichen Verhältnissen innerhalb der politischen Ökologie am Beispiel der Wasserthematik verhandelt. Insbesondere Linton (2010) hat mit seinem Buch „What is Water?" die Diskussionen über das ontologische Verständnis von Wasser vorangetrieben. Dabei zeigt er auf, wie das hegemoniale, essentialistische Konzept des „modernen Wassers“ (modern water) Wasser als eine abstrakte, isomorphe und quantitativ messbare Einheit erzeugt, die auf die Formel $\mathrm{H}_{2} \mathrm{O}$ reduziert werden kann und als solche in einem globalen Wasserkreislauf zirkuliert (ebd.:14). Demgegenüber stellt er das Konzept der sozialen Natur von Wasser. Dabei produziert Gesellschaft Wasser über soziale Prozesse und Praktiken, sodass jegliches Wasser bereits mit Ideen, Bedeutungen, Werten und Potentialen aufgeladen ist (ebd.:5). Jedoch zeigt sich allein an der Tatsache, dass unser Körper zu einem Großteil aus Wasser besteht, dass die Grenzen zwischen Wasser und Gesellschaft, zwischen einem Innen und einem Außen, zwischen Subjekt und Objekt „flüssig“ sind. Folglich produzieren Menschen nicht nur Wasser als abstraktes Gebilde, sondern sind aufgrund ihrer Körperlichkeit immer schon Teil dieses Produktionsprozesses. In den vielfältigen Prozessen der Herstellung von Wasser stellen wir letztendlich immer auch uns selbst her: „We mix language, gods, bodies, and thought with water to produce the worlds and the selves we inhabit" (ebd.:3).

Jedoch wird Wasser nicht allein über soziale Praktiken hergestellt. Vielmehr sind neben den Prozessen der Bedeutungszuschreibungen, Repräsentationsmechanismen und Machtbeziehungen auch Kräfte wie Klima, Jahreszeiten, Luftdruck, Geomorphologie und zahllose andere nichtmenschliche Lebewesen in die Ko-Konstitution des Wassers involviert (ebd.:34). Wichtig dabei ist, dass die Repräsentationen von Wasser nicht als soziale Konstruktionen den physikalischen Eigenschaften des Wassers als wahre Wesenszüge gegenübergestellt werden: „Physical properties, as well as meanings and representations, are not essential to water, nor are they merely imposed from what is beyond. [... ] Nor can we impose any identity or behaviour on water in the manner of a pure social construction. Our constructions - as well as our material productions - of water must be constrained by the reality of water's myriad engagements" (ebd.:34-35).

Gemeinsam mit Bakker (2002), Budds (2008) oder Kaika (2005) versteht Jamie Linton den globalen Wasserkreislauf nicht als gegebene Tatsache der materiellen Welt, die wissenschaftlich beschrieben werden kann, sondern als Konzept, in dem die physikalischen Eigenschaften des Wassers, die hydrologischen Besonderheiten der Orte, an denen das Konzept entstanden ist, die hegemonialen Vorstellungen von 
Natur, der Forschungsstand der Hydrologie und die vorherrschende Nutzungsart des Wassers verinnerlicht sind (Linton, 2010:35; Bouleau, 2014:249). Dabei verändern sich im Laufe der Zeit die Vorstellungen über den Wasserkreislauf in Abhängigkeit von den kulturellen, sozialen und geographischen Verhältnissen. Diese Veränderungen sind jedoch nicht beliebig, sondern werden notwendigerweise von den biophysischen Eigenschaften des Wassers (Schwerkraft, Veränderung der Aggregatszustände etc.) beschränkt (Linton, 2010:35). Der Wasserkreislauf wird daher als hydrosozialer Kreislauf verstanden, durch den Wasser über interne sozioökologische Prozesse ständig als Hybrid aus materiellen und diskursiven Elementen hergestellt wird, wodurch neue vergesellschaftete Naturen (socio-natures) entstehen (Linton und Budds, 2014; Swyngedouw, 2015:21).

Den Begriff der socio-natures hat vor allem Erik Swyngedouw geprägt. In seinen zahlreichen Arbeiten beschreibt er, wie Wasser durch Landschaften, technische Arrangements und Städte fließt, dabei Orte und Menschen, die menschliche und die nicht-menschliche Sphäre miteinander verknüpft und neue hydro-soziale Landschaften (hydro-social landscapes bzw. hydroscapes) herstellt (Swyngedouw, 1999:445, 2015:21). Auch Swyngedouw macht deutlich, dass Wasser nicht bloß eine passive Materie darstellt. Vielmehr betont er das Handeln (acting) von Wasser, das über eine bestimmte Zusammensetzung verschiedener Elemente - technologische Systeme, akademisches Wissen, politische Institutionen, wirtschaftliche Kräfte, soziale Praktiken, politische Netzwerke und Regime - vermittelt wird (Swyngedouw, 2015:28). Verändert sich diese Zusammensetzung, so verändern sich auch die Möglichkeiten des materiellen Handelns des Wassers (ebd.:29). Ein solches Handeln ist jedoch weder vollständig vorhersehbar noch kontrollierbar. Jegliche Versuche, die Natur zu beherrschen und sie gänzlich ingenieurstechnisch zu kontrollieren, sind also unweigerlich zum Scheitern verurteilt. Somit wird es immer einen Rest geben, der der kompletten Vereinnahmung widersteht: „Cyborgs and hybrids are imperfect creatures and cannot be but so. The attempts to engineer, master, and control the hydrosocial cycle, to keep ,nature on a leash', are never complete, never fulfill fully their Promethean promise. The actants in hydro-social networks often [...] push beyond the bounds in which they are imagined to dwell, and behave in strange and often unpredicted, if not unpredictable, ways“ (ebd.).

Die besondere Rolle von materiellen Infrastrukturen wird vor allem auch in der urbanen politischen Ökologie diskutiert. Dabei wird ihr Verhältnis zu Ressource-, Wasserund Energieflüssen, sowie ihre Einbettung in kulturelle und polit-ökonomische Prozesse thematisiert. Zentraler Bezugspunkt ist dabei die Metapher eines urbanen Stoffwechsels (urban metabolism), wobei insbesondere Gandy (2004) auf die unterschiedliche Verwendungsweise der Metapher hinweist. Während in einer funktionalistischen Konzeption die Stadt als Körper und der Stoffwechsel mit der Natur als eine Art bio-physischer Austauschprozess zwischen zwei un- abhängigen Sphären verstanden wird, wird in einer neomarxistischen Interpretation das dialektische Verhältnis und die wechselseitige Konstitution von natürlichen und sozialen Prozessen betont (ebd.:364). In diesem Sinne kann untersucht werden, wie bspw. Urbanisierungsprozesse, Produktionsbedingungen, kulturelle Ausdrucksformen, Machtverhältnisse usw. die sozialökologischen Interaktionen in Städten formen und durch diese geformt werden (Monstadt, 2009:1933). Die materiellen Bedingungen können dabei als unabdingbare Voraussetzung für den urbanen Stoffwechsel angesehen werden und werden gleichzeitig über diesen in eine neue, warenförmig organisierte urbane Natur (Nahrungsmittel, Energie, Trinkwasser) transformiert (Gandy, 2004:374). ,,[C]ities are built in and with nature and [...] they produce nature“ (Monstadt, 2009:1933).

In dieser Sichtweise sind Infrastruktureinrichtungen wie die Wasserinfrastruktur sozioökologische Systeme, durch die Natur genutzt und gleichzeitig verwandelt wird. In Anlehnung an die ANT können Wassernetzwerke als aktive Akteure der Raumproduktion verstanden werden (Gandy, 2004:374), durch die Natur, sowohl in Form von intendierten Ergebnissen (Trinkwasserversorgung) als auch in Form von unintendierten Folgen (Verunreinigungen, Flächenverbrauch, Lecks usw.) neu hergestellt wird (Monstadt, 2009:1933). Gleichzeitig nehmen sie dabei immer auch eine aktive Rolle bei der Produktion von urbanem Leben und urbaner Kultur ein (Gandy, 2004:374).

In diesem komplexen Geflecht von Mensch-NaturBeziehungen spielen neben Diskursen, Materialität und Infrastruktureinrichtungen auch gesetzliche Bestimmungen, wasserbezogene Praktiken und die Herausbildung von Subjektpositionen eine wichtige Rolle. Erst im Zusammenspiel der unterschiedlichen Dimensionen werden spezifische gesellschaftliche Naturverhältnisse hervorgebracht und transformiert. Gegenüber den bereits beschriebenen Konzepten soll in diesem Aufsatz der Beitrag einer Dispositivanalyse für das Verständnis der Mehrdimensionalität gesellschaftlicher Naturverhältnisse, ihre diskursive und materielle Ordnung und deren wechselseitigen Bedingungsverhältnisse herausgearbeitet werden.

\section{Dispositivanalyse}

Ähnlich wie der Begriff des Diskurses ist auch der Begriff des Dispositives in der französischen Sprache ein fest verankerter Ausdruck. Darunter kann eine bestimmte Konstellation von Maßnahmen und Objekten verstanden werden, die zu einem spezifischen Zweck miteinander in Verbindung stehen und dabei jedoch nur als Ganzes funktionieren. Angelehnt ist die Dispositivanalyse insbesondere an die Überlegungen von Michel Foucault, der ein Dispositiv als ,ein entschieden heterogenes Ensemble“ definiert, „,das Diskurse, Institutionen, architekturale Einrichtungen, reglementierende Entscheidungen, Gesetze, administrative Maßnahmen, wissen- 
schaftliche Aussagen, philosophische, moralische oder philanthropische Lehrsätze, kurz: Gesagtes ebensowohl wie Ungesagtes umfasst. Soweit die Elemente des Dispositivs. Das Dispositiv selbst ist das Netz, das zwischen diesen Elementen geknüpft werden kann" (Foucault, 1978:119-120).

Beispielsweise könnte eine Alarmanlage mitsamt ihrer technischen Vorrichtungen (Sensoren, Kabel, Apparaturen), dem in ihr vorhandenen technischen Wissen, den eingeschriebenen Handlungsweisen (An- und Ausschalten mit Hilfe eines Geheimcodes), den rechtlichen Rahmenbedingungen usw. als Dispositiv beschrieben werden (Keller, 2007:50; Bührmann und Schneider, 2008:51-52). Demzufolge werden Dispositive oftmals als ,materielle und ideelle Infrastruktur" (Keller, 2008:235) bezeichnet, über die Diskurse stabilisiert werden. Eine solche Infrastruktur-Metapher birgt jedoch die Gefahr der Verkürzung in sich. Ein Dispositiv ist nicht als ein Nebeneinander einzelner Elemente und eine Dispositivanalyse nicht als bloße Bestandsaufnahme der beteiligten Einheiten zu verstehen. Vielmehr ist das Dispositiv das Netz, das zwischen ihnen geknüpft werden kann, das Dazwischen, die Verbindungen, die den Zusammenhalt zwischen den Elementen ausmachen. Gleichzeitig sind diese Verbindungen jedoch nicht neutral, sondern üben immer auch eine machtstrategische Funktion aus, indem über sie bestimmte (Wissens)Ordnungen hervorgebracht und gestützt werden (Bührmann und Schneider, 2008:52-53).

Eine explizite Methodologie zur Durchführung einer Dispositivanalyse gibt es nicht (Jäger, 2001:72). Foucault selbst hat in seinen Schriften Überlegungen zu einem Sicherheitsbzw. Sexualitätsdispositiv angestellt. Bührmann und Schneider (2008) geben in ihrem Buch „Vom Diskurs zum Dispositiv" anhand zweier Beispiele (Geschlechterdispositiv, Sterbe-/Todesdispositiv) Hinweise zur methodischen Umsetzung einer Dispositivanalyse. Jedoch bleibt die Durchführung einer solchen Untersuchung mit der Einbeziehung des umfangreichen, unterschiedlichen und oft widerständigen Materials aus der empirischen Forschungspraxis eine beträchtliche Herausforderung.

Die Hauptfunktion eines Dispositivs sieht Foucault darin, ,zu einem gegebenen historischen Zeitpunkt [...] auf einen Notstand (urgence) zu antworten. Das Dispositiv hat also eine vorwiegend strategische Funktion" (Foucault, 1978:120). Ein solcher Notstand - wie etwa eine Dürre - ist jedoch nicht objektiv gegeben sondern wird diskursiv hergestellt. Der Ausgangspunkt einer Dispositivanalyse kann somit die Untersuchung der Herstellung von Problemlagen mit Hilfe einer Diskursanalyse darstellen. Zentrales Moment dabei ist, dass die soziale und materielle Welt in Diskursen nicht einfach nur reflektiert, sondern auf eine spezifische Art und Weise hergestellt wird (Foucault, 1973:74; Keller, 2008:63). Demnach kann anhand einer Diskursanalyse herausgearbeitet werden, was als Problem wahrgenommen und benannt wird, welche Lösungsstrategien sich daraus ergeben, welche Subjektpositionen zur Verfügung gestellt und welche Praktiken legitimiert werden.

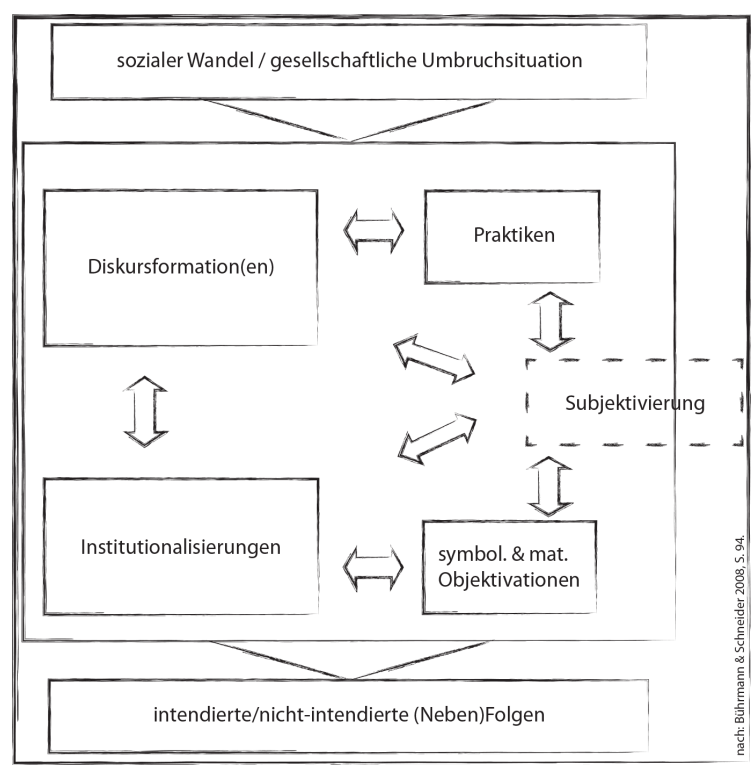

Abb. 1. Dimensionen einer Dispositivanalyse.

Die strategische Funktion des Dispositivs besteht laut Foucault nun darin, über ein Set von Diskursen, Praktiken, Institutionen und Materialisierungen Antworten auf eine diskursiv hergestellte Problemlage zur Verfügung zu stellen. Zwar wird ein Dispositiv als Ensemble verstanden, dessen Elemente untrennbar miteinander in Verbindung stehen. Da das Dispositiv jedoch nicht als amorphe Einheit oder als untrennbares Hybrid, sondern als Netz zwischen verschiedenen Elementen benannt wird, können diese in ihrer Besonderheit beschrieben und gleichzeitig die Verbindungen zwischen ihnen untersucht werden. Aufgrund der Vielschichtigkeit der Dimensionen eines Dispositivs können neben einer Diskusanalyse verschiedene ethnographische Methoden, die Analyse institutioneller Arrangements, die Beschreibung und Kartierung von Raumstrukturen etc. als Untersuchungsmethoden in die Analyse integriert werden. Insofern stellt eine Dispositivanalyse einen „triangulierenden Forschungsprozess“ (Bührmann und Schneider, 2008:92) dar.

In Anlehnung an die Darstellungsweise von Andrea Bührmann und Werner Schneider (Bührmann und Schneider, 2008:94) werden in Abb. 1 die Elemente eines Dispositivs dargestellt. Auch wenn der Fokus einer Dispositivanalyse auf den unterschiedlichen Beziehungen und gegenseitigen Bedingungsverhältnissen liegt, sollen dadurch keine kausalen Ursachen-Wirkungszusammenhänge aufgespürt werden. Es geht eher um das „Spiel von Positionswechsel und Funktionsveränderungen“ (Foucault, 1978:120), das in seiner Vielfalt sicherlich nicht vollständig erfasst, jedoch zumindest in den Blick genommen werden kann. Das Dispositiv als formierendes Netz mit machtstrategischer Funktion zu begreifen bedeutet, die Machtwirkungen in den Beziehungen aufzuspüren (Bührmann und Schneider, 2008:52). In diesem Verständnis ist Macht nicht etwas, was besessen und einsei- 
tig ausgeübt werden kann, sondern etwas, das zwischen den Elementen wirkt, das kanalisiert, erschwert und ermöglicht, ,was sich von unzähligen Punkten aus und im Spiel ungleicher Beziehungen vollzieht" (Foucault, 1983:115). Demzufolge ist die Analyse der gegenseitigen Wechselverhältnisse und ihrer Wirkungsweisen innerhalb eines Dispositivs immer auch eine Analyse von Machtverhältnissen. So ist danach zu fragen, wie bestimmte Diskursanordnungen dominant werden, wie diese über Institutionalisierungen auf Dauer gestellt werden, welche Subjektpositionen mit Sprech- und Handlungsmacht ausgestattet werden, wie sich Diskurse in den Raum einschreiben und materialisieren, welche Praktiken hervorgebracht werden und welche Rückwirkungen sich daraus wiederum für die Wissensordnung ergeben.

Foucault interessierte sich insbesondere auch für die $\mathrm{Zu}$ sammenhänge zwischen materiellen Anordnungen und deren Auswirkungen auf die Handlungsweisen von Akteuren. In „Überwachen und Strafen“ geht er in Anlehnung an das Panoptikum von Jeremy Bentham auf die Gefängnisarchitektur ein, die ein bestimmtes Verhalten der Individuen konditioniert und Überwachungspraktiken in Praktiken der Selbstkontrolle verwandelt. Dabei wird die Machtwirkung nicht allein auf die Handlungen einzelner Personen zurückgeführt. Vielmehr wird angenommen, dass sie von der mit Bedeutung aufgeladenen Anordnung der Dinge ausgeht: „,Das Prinzip der Macht liegt weniger in einer Person als vielmehr in einer konzertierten Anordnung von Körpern, Oberflächen, Lichtern und Blicken“ (Foucault, 1977:259). Gebaute Umwelt kann demnach immer auch als Vergegenständlichung von sozialen Beziehungen gelesen werden, über die Macht ausgeübt wird (Bührmann und Schneider, 2008:103; Wissen, 2011:105 f.).

Eine solche Machtwirkung, die aus dem Zusammenwirken der einzelnen Elemente des Dispositivs entsteht, kann als Agency beschrieben werden. Dabei wird weder davon ausgegangen, dass die Dinge an sich handeln, noch dass Diskurse bestimmte Praktiken determinieren. Jedoch können die Subjekte nicht länger als alleinige Urheber ihrer Handlungsweisen verstanden werden. Vielmehr geht die Handlung aus dem untrennbaren Zusammenspiel zwischen Diskursen, Subjekten und Dingen erst hervor. Bestimmte materielle Anordnungen üben dabei eine stärkere disziplinierende Wirkung auf die Handlungsweisen der Akteure aus als andere. Indem im Zusammenspiel der Materialität der Dinge mit institutionellen Vorgaben und dem Vorhandensein bestimmter Subjektpositionen einige Verhaltensweisen erleichtert, andere erschwert werden, werden Handlungen kanalisiert und geleitet. Somit lässt sich konstatieren, dass im Dispositiv Handlungsskripte eingeschrieben sind, die bestimmte Verhaltens- und Gebrauchsweisen zumindest wahrscheinlicher machen (vgl. Bührmann und Schneider, 2008:103).

In diesem Sinne bietet die Dispositivanalyse einen konzeptionellen Rahmen, um die unterschiedlichen Dimensionen gesellschaftlicher Naturverhältnisse ansprechen und in ihren wechselseitigen Bedingungsverhältnissen analysieren zu können. Doch gerade aufgrund der Komplexität der Verhältnisse und der Vielschichtigkeit der Wechselbeziehungen stellt die Durchführung einer solchen Analyse eine erhebliche Herausforderung für empirisch angelegte Arbeiten dar. Im Folgenden soll anhand des Phänomens der Dürre als Teil gesellschaftlicher Naturverhältnisse im Nordosten Brasiliens ausgelotet werden, inwieweit eine Dispositivanalyse dabei helfen kann, die Beziehungen zwischen den einzelnen Elementen herauszuarbeiten und die darin eingeschriebenen Machtverhältnisse zu benennen. Der Schwerpunkt soll dabei vor allem auf dem Zusammenhang zwischen Diskursen und Materialisierungen liegen. Auf die weiteren Elemente des Dispositivs (Institutionalisierungen, Subjektivationen, Praktiken) kann innerhalb dieses Artikels nicht näher eingegangen, sondern lediglich auf einige Anknüpfungspunkte kurz verwiesen werden (ausführlicher: Schmitt, 2013).

\section{Fallbeispiel: Das Dispositiv der Dürre im Nordosten Brasiliens}

Für die empirische Anwendung einer Dispositivanalyse wurden die Dürreverhältnisse im Nordosten Brasiliens, genauer in der Region Baixo Jaguaribe im Bundesstaat Ceará untersucht. Der Nordosten Brasiliens kann als besonders emblematisch für eine räumliche Einheit angesehen werden, die über spezifische gesellschaftliche Naturverhältnisse - insbesondere den Dürreverhältnissen - als abgrenzbare Region konstituiert wird (Schmitt, 2013:104 ff.). Niederschläge, bzw. das Ausbleiben von Niederschlägen werden dabei nicht allein als Grundlage für die Landwirtschaft und die Wasserversorgung der Bevölkerung oder als Standortfaktor für die Tourismusindustrie verhandelt. Vielmehr werden sie als integraler Bestandteil der Geschichte des Nordostens und des Selbst- und Fremdverständnisses der „Nordestinos“ verstanden. Sie spiegeln sich in der Vielzahl an Programmen, Institutionen und Organisationen der Dürrebekämpfung wider und sind aus den Diskursen über Fortschritt und Entwicklungsmodelle nicht wegzudenken. Insbesondere die als „Große Dürre“ bezeichnete Dürreperiode zwischen 18771879, der allein im Bundesstaat Ceará Schätzungen zufolge fast die Hälfte der Bevölkerung zum Opfer fiel (Taddei, 2005:113; Carvalho, 1988:195), wurde zu einem Kristallisationspunkt der Entstehung des Nordostens als „Armenhaus" Brasiliens. Durch dieses Ereignis wurde die Dürre zur unhinterfragbaren Realität des Nordostens, wodurch sich die gesellschaftlichen Probleme in Probleme eines spezifischen Raumes verwandelten (Albuquerque, 1999:199; Bartelt, 2001:343).

Als konkrete Untersuchungsregion diente die Region Baixo Jaguaribe im Nordosten des Bundesstaates Ceará (s. Abb. 2), da hier seit den 1990er Jahren tiefgreifenden Veränderungen der gesellschaftlichen Naturverhältnisse in Bezug auf Wasser stattgefunden haben. Zum einen wurde in Ceará bereits 1992 ein Integriertes Wasserressourcen-Management 
(IWRM) gesetzlich verankert, wodurch dem Bundesstaat eine Vorreiterrolle in Sachen integrierter Ressourcenpolitik zukam. Zum anderen kam es speziell in der Region Baixo Jaguaribe zu einem großflächigen Ausbau der Wasserinfrastruktur und zu einer massiven Expansion der Flächen der Bewässerungslandwirtschaft und Shrimpszucht für den Export (IPECE, 2007b). Dies führte zu räumlichen und sozialen Umstrukturierungsprozessen, die mit einer Erhöhung der Konflikte um die Nutzung von und den Zugang zu Land und Wasser und mit einer verstärkten Mobilisierung von Widerstand einhergingen. Aufgrund dieser Entwicklungen wurde die Region von Elias und Sampaio (2002) auch als Beispielregion für eine exkludierende Modernisierung bezeichnet.

Werden die Niederschlagsverhältnisse der Region nun nicht als externe natürliche Bedingungen, sondern als Bestandteil eines (sozio-materiellen) Dispositivs verstanden, kann deren sozialer Konstruktionscharakter aufgezeigt werden, ohne jedoch die physisch-materiellen Bedingungen auszublenden oder in eine dualistische Deutungsweise zu verfallen. Dabei kann aufgezeigt werden, wie das Dürredispositiv sowohl über messbare, materielle Bedingungen, als auch über deren Interpretation und diskursive Verhandlung und den sich gegenseitig bedingenden Wechselwirkungen konstituiert wird. Das Dispositiv entfaltet sich, indem sich Diskurse über Dürre in institutionelle Arrangements einschreiben, sich in der gebauten Umwelt manifestieren, bestimmte Subjektpositionen hervorbringen und verschiedene Handlungsweisen ermöglichen oder erschweren. Dabei wird das Dispositiv über die Niederschlagsverhältnisse gestärkt und weiter verankert. Gleichzeitig können jedoch Ereignisse wie etwa Dürren oder Überschwemmungen auch dazu führen, dass die bestehende Diskursordnung in Frage gestellt wird und neu geordnet werden muss (Mattisek und Wiertz, 2014:158).

Ausgangspunkt der Analyse des Dürredispositivs in der Region Baixo Jaguaribe stellt eine Analyse des Dürrediskurses und der Konstitution der spezifischen Problemlage sowie den darin eingeschriebenen Lösungsansätzen dar. Darüber hinaus werden die verschiedenen Verknüpfungen mit den unterschiedlichen Elementen des Dispositivs angedeutet, um dann insbesondere auf die Materialisierung des Diskurses und die Diskursivität der materiellen Ordnung einzugehen. Im Anschluss daran soll der Frage nachgegangen werden, welche Effekte über das Dispositiv hervorgebracht werden und welche Widerstände und Verschiebungen sich dabei ergeben können.

\subsection{Der Dürrediskurs}

Für die Freilegung von Wissensordnungen und die Benennung von Strukturen und Regelhaftigkeiten des Dürrediskurses in Ceará wurde eine Diskursanalyse vorgenommen. Ausgehend von der Annahme, dass (Massen)Medien als ,zentrale Arenen der gesellschaftlichen Wirklichkeitskonstruktion“ (Keller, 2008:79) verstanden werden können, über die bestimmte Themen bzw. Problemkomplexe gerahmt werden (ebd.), wurden 340 Artikel der größten Tageszeitung in Ceará (Diário do Nordeste) aus den Jahren 2008-2012 analysiert. Darüber hinaus wurde eine sechsstündige Senatsdebatte, in der Gegner*innen und Befürworter*innen des Ableitungsprojektes des Rio São Francisco - dem wichtigsten und symbolträchtigsten Projekt zur Bekämpfung der Dürre im Nordosten Brasiliens - zu Wort kamen, transkribiert und analysiert. Und schließlich wurden noch drei Reden des ehemaligen Präsidenten Inácio Lula da Silva, als herausragende Sprechposition innerhalb des Diskurses um die Bearbeitung der Dürre, in die Analyse mit einbezogen.

Anhand der Analyse des Diskurses über die Dürre in Ceará wurde in erster Linie deutlich, dass nach wie vor der physische Wassermangel als zentrales Problem der Region verhandelt wird. Durch diese hegemoniale Deutungsweise der Dürreverhältnisse wird die Erhöhung des Wasserangebots durch den Ausbau der Wasserinfrastruktur (Staudämme, Brunnen, Flussableitungen etc.) zur unmittelbar notwendigen Lösungsstrategie erhoben. Dabei wird eine direkte Kausalbeziehung zwischen den natürlichen Bedingungen (v.a. geringe Niederschläge) und den gesellschaftlichen Verhältnissen (meist als Armut und Rückständigkeit bezeichnet) hergestellt. Dies hat zur Folge, dass jegliche Mechanismen der sozialen Vermittlung - wie etwa die Regelung des $\mathrm{Zu}$ gangs zu Wasser, Besitzverhältnisse, Machtverhältnisse, historische Entwicklungen etc. - ausgeblendet und somit unsichtbar gemacht werden. Über die Darstellungsweise der besonderen Dringlichkeit und des riesigen Ausmaßes des Problems erscheinen Großprojekte, mit deren Hilfe besonders viel Wasser gespeichert oder transportiert werden kann, als unabdingbar. Mögliche dezentrale, kleinstrukturierte Projekte der Wasserversorgung oder Ansätze einer Landwirtschaft im Einklang mit den semi-ariden Bedingungen der Region (convivência com o semi-árido) gelten in Anbetracht der Dimension des Problems als ineffektiv und werden über den Diskurs als adäquate Lösungen ausgeschlossen.

Gleichzeitig ist der Dürrediskurs in einen globalen Diskurs um Knappheit, Nachhaltigkeit, Ressourceneffizienz und Wirtschaftlichkeit eingebettet. In Bezug auf den Umgang mit Wasser wird dabei vor allem auf die „Dublin-Prinzipien“ verwiesen, die 1992 auf der Konferenz über Wasser und Umwelt (ICWE) verabschiedet wurden und eine dominante Stellung innerhalb des globalen Wasserdiskurses einnehmen. In den Prinzipien wird Wasser als endliche Ressource mit ökonomischem Wert definiert, die über partizipative ManagementMethoden verwaltet werden soll (ICWE, 1992:4). Aus dieser Logik wird eine gewinnmaximierende Inwertsetzung der knappen Ressource Wasser, insbesondere über den Ausbau einer exportorientierten Bewässerungslandwirtschaft, als angemessene und legitime Lösungsstrategie abgeleitet.

\subsection{Das Aufspannen des Netzes}

Der hier nur sehr skizzenhaft vorgestellte Dürrediskurs manifestiert sich auch in den übrigen Elementen des Dürredis- 
positivs und wird über diese verfestigt. Dies ist jedoch nicht als einseitige Einwirkung auf Gesetze und Subjektpositionen oder als Determinierung von Praktiken zu verstehen. Vielmehr geht es um sich wechselseitig verstärkende Prozesse des Einschreibens und Rückwirkens, des Legitimierens und Bestätigens, des Ermöglichens und Verstärkens. So wurde beispielsweise in Ceará als zweitem Bundesstaat Brasiliens ein Integriertes Wasserressourcen Management gesetzlich festgeschrieben. Darin wurden Wassereinzugsgebiete, Wassertarife und Wasserkomitees als zentrale Instrumente der staatlichen Wasserpolitik institutionell verankert. Durch die Festlegung von Wassereinzugsgebieten als neue, administrative Einheiten kam es zu einer räumlichen Reorganisation des Wassermanagements und zu einer Reterritorialisierung von Entscheidungsstrukturen. Darüber hinaus wurden Wassertarife für nicht behandeltes Oberflächenwasser eingeführt, wodurch die in der Region betriebene wasserintensive Reisproduktion, die vor allem auf die lokalen Bedürfnisse ausgerichtet war, unrentabel wurde. Gleichzeitig breitete sich seit Ende der 1990er Jahre aufgrund hoher Gewinnmargen und zahlreicher Vergünstigungen in einigen Regionen eine exportorientierte Obstproduktion auf Bewässerungsbasis aus. Für die Umsetzung eines partizipativen WasserressourcenManagement wurden regionale Wasserkomitees ins Leben gerufen, in denen in erster Linie über die Abflussmenge von Staudämmen abgestimmt wird. Auch wenn die Erfahrungen der letzten Jahre gezeigt haben, dass über diese Art der Beteiligungspolitik grundsätzliche Änderungen der Wasserpolitik und eine Neuregelung von Besitzverhältnissen und Zugangsrechten eher verhindert als möglich werden, so erhöhte die Einbettung der Wasserpolitik in einen Diskurs von Teilhabe und Mitbestimmung die Legitimation der staatlichen Politik in Ceará (Schmitt, 2016).

Durch die Einführung eines partizipativen Wassermanagements werden Praktiken der Kooperation und des Ausgleiches gestärkt, während radikale Gegenentwürfe zum vorherrschenden Entwicklungsmodell und widerständiges Verhalten marginalisiert werden. Über den Diskurs des Ressourcenmanagements werden die verschiedenen Akteure als sogenannte Stakeholder angerufen, d.h. in erster Linie als Interessensvertreter*innen, die über einen Konsens die knappe Ressource Wasser verwalten. Unterschiedliche Positionen werden als Stakeholder in die staatliche Wasserpolitik kooperativ eingebunden, während widerständige Positionen, wie etwa die der Landlosenbewegung MST (Movimento dos Trabalhadores Rurais Sem Terra) zunehmend kriminalisiert werden. Gleichzeitig wird der Staat als zentrale Position des Wassermanagements inszeniert, dessen Vertreter*innen über Insiderwissen und Handlungsmacht verfügen. Dabei stellt ein naturwissenschaftlich-technisches Wissen das entscheidende Zugangskriterium für ein legitimes Sprechen innerhalb des Diskurses dar, während lokales Wissen, Erfahrungswissen oder indigenes Wissen tendenziell marginalisiert wird. Schließlich ergibt eine Analyse der Sprechpositionen, dass das Sprechen über die Dürre im Nordosten und die Suche nach möglichen Lösungsansätzen nach wie vor eine männlich dominierte Position darstellt.

\subsection{Wasserinfrastruktur als symbolische und materielle Objektivation}

An den Infrastrukturen der Wasserversorgung und speicherung lässt sich das Wechselverhältnis zwischen Diskursivität und Materialität besonders anschaulich nachvollziehen. Dabei kann sowohl danach gefragt werden, wie sich Diskurse und Machtverhältnisse in die Umwelt einschreiben, als auch, welche Wirkmacht Infrastruktureinrichtungen auf Wahrnehmungen, Diskurse und Praktiken ausüben und welche Eigenständigkeit von Materialität dadurch zum Vorschein kommt. Gerade in einer semi-ariden Region bildet die Wasserinfrastruktur eine zentrale Schnittstelle gesellschaftlicher Naturverhältnisse, über die die Beziehungen zwischen Natur und Gesellschaft organisiert und vermittelt werden. Sie kann sowohl als physisch-materielle Grundlage für die Aneignung und Transformation von Natur als auch als Resultat gesellschaftlicher Aushandlungsprozesse und symbolischer Ausdruck von Kräfteverhältnissen gelesen werden (vgl. Swyngedouw, 2015; Bakker, 2003; Wissen, 2011:107). Über die Infrastruktursysteme werden insbesondere die Zugriffs- und Aneignungsmöglichkeiten von Wasser verändert. Mithilfe von Stauseen, Kanälen, Pumpen und Leitungen kann Wasser gezielt als Produktionsfaktor in der Bewässerungslandwirtschaft eingesetzt werden. Über Schleusen kann die Verteilung gesteuert und über Wasserzähler der Wasserverbrauch kontrolliert und die Verfügbarkeit monetär geregelt werden. Gemäß gesellschaftlicher Imaginationen und wissenschaftlich hergestelltem Wissen wird Wasser mit Hilfe von Infrastruktureinrichtungen in eine isolierte Einheit verwandelt, die wiederum transportiert, zentriert, gespeichert und genutzt werden kann; die evaporiert, versickert, versalzt und verschmutzt; die gemessen, bewertet und verkauft werden kann. Dadurch wird Wasser in eine Ressource und somit in kommodifizierte Natur verwandelt (Kaika, 2008:91).

\subsubsection{Materialisierung von Diskursen}

Betrachtet man die Dürreverhältnisse als Dispositiv, dann stellen die Wasserinfrastrukturprojekte keine isolierten Maßnahmen zur Bekämpfung der Dürre dar, sondern können im Zusammenspiel mit den weiteren Dimensionen als konstituierendes Element der Dürreverhältnisse verstanden werden. Die Ausweitung der Wasserspeicherkapazität als Lösungsstrategie materialisiert sich in riesigen Staumauern und kilometerlangen Kanalwänden und schreibt sich über diese in den Raum ein. Insbesondere im Nordosten des Bundesstaates Ceará wurden in den letzten Jahren vermehrt Großprojekte der Wasserinfrastruktur umgesetzt. Mit dem Bau des Castanhão mit einer Speicherkapazität von $6,7 \mathrm{Mrd} . \mathrm{m}^{3}$ wurde in dieser Region der zweitgrößte Stausee des Nordosten Brasiliens aufgestaut. Darüber hinaus wird hier das Kanal- 


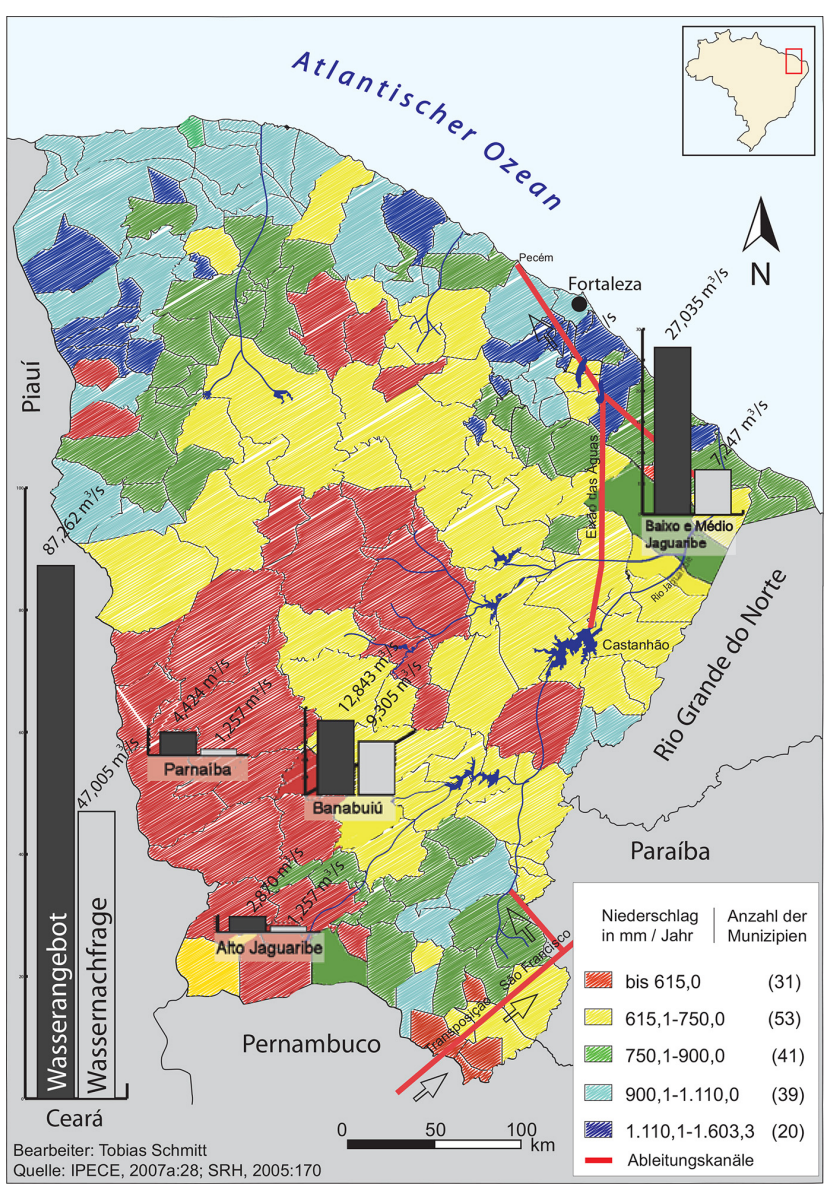

Abb. 2. Jahresdurchschnittsniederschläge (1974-2003) und Wasserbilanz ausgewählter Wassereinzugsgebiete in Ceará.

system Eixão das Águas (Wasserachse) mit insgesamt 255 km Wasserkanälen, Pumpstationen, Tunneln und Wasserleitungen errichtet (ADECE, 2015). In dieses System wird auch das Wasser aus dem Ableitungsprojekt des Rio São Francisco, dem größten und umstrittensten Flussableitungsprojekt Brasiliens, geleitet.

Legitimiert wird der Bau solcher Großprojekte über die Niederschlagsverhältnisse, die als objektive und messbare Fakten die naturräumliche Benachteiligung der Region belegen sollen. Betrachtet man jedoch die durchschnittlichen Jahresniederschläge nicht auf bundesstaatlicher Ebene, sondern differenziert nach den einzelnen Wassereinzugsgebieten (Abb. 2), so wird deutlich, dass Dürre nicht als homogenes Phänomen, das den gesamten Bundesstaat gleich stark betrifft, dargestellt werden kann. Zieht man die - von staatlichen Behörden erhobene - Wasserbilanz hinzu, zeigt sich noch viel deutlicher, dass vor allem der Südwesten des Bundesstaates eine geringe Wasserverfügbarkeit aufweist, während im Nordosten eine vergleichsweise hohe Wassersicherheit verzeichnet werden kann.

Allein an diesem Beispiel wird deutlich, dass keine direkten Kausalzusammenhänge zwischen natürlichen Bedin- gungen, hegemonialem Wissen und gesellschaftlichen Praktiken (Ausbau der Infrastruktur) angenommen werden können. Vielmehr werden die materiellen Gegebenheiten über Diskurse in Kausalzusammenhänge eingebettet, interpretiert und vermittelt, wobei spezifische Machtverhältnisse zum Tragen kommen. Durch die Herstellung des Nordosten Brasiliens als homogenen, von der Dürre betroffenen Raum, können alle Wasserinfrastrukturprojekte der Region als Maßnahmen der Dürrebekämpfung gerahmt werden. Verschnitten mit den Diskurssträngen einer effizienten und gewinnbringenden Wassernutzung führt der spezifische Ausbau der Wasserinfrastruktur zu einer gezielten Inwertsetzung bestimmter Räume für die exportorientierte Agrarindustrie. So soll über den einseitigen Ausbau der Wasserinfrastruktur die exportorientierte Bewässerungslandwirtschaft und Shrimpszucht in der Region Baixo Jaguaribe weiter ausgebaut, die Wasserversorgung der Metropolitanregion Fortaleza (mit über 3,5 Mio. Einwohner*innen) gesichert und der Ausbau des Industriehafens Pecém, inklusive der Ansiedlung von mehreren Unternehmen mit enorm großem Wasserbedarf (Stahlindustrie, Kohlekraftwerk, Zementfabriken etc.) ermöglicht werden (ADECE, 2013).

Der scheinbare Widerspruch zwischen dem Wassermangel im Südwesten und dem Ausbau der Wasserinfrastruktur im Nordosten des Bundesstaates kann somit nur über das dahinter liegende Entwicklungsmodell und die darin eingeschriebenen Machtverhältnisse erklärt werden. Der Ausbau der Wasserinfrastruktur in Ceará kann dabei nicht als unmittelbare Antwort auf die Niederschlagsverhältnisse begriffen werden. Vielmehr ist sie manifester Ausdruck einer hegemonialen Diskursordnung, in der politische und ökonomische Interessen und Machtverhältnisse verwoben sind. Das zur knappen Ressource erhobene Wasser wird insbesondere in denjenigen Regionen konzentriert, in denen es effektiv eingesetzt werden kann und hohe Gewinne in Aussicht stehen.

\subsubsection{Materialität als Verstetigung von Machtverhältnissen}

Über die spezifische Materialität der Wasserinfrastruktur und ihre territoriale Verbreitung schreiben sich folglich Machtverhältnisse in die Umwelt ein und werden auf Dauer gestellt. Ein solches Einschreiben von Diskursen und Machtverhältnissen in die gebaute Umwelt kann jedoch weder als Automatismus noch als linearer Prozess verstanden werden. Vielmehr ist der Prozess der Materialisierung geprägt von gesellschaftlichen Auseinandersetzungen und ständigen Aushandlungsprozessen. Wo welcher Staudamm mit welcher Größe und zu welchem Zweck gebaut wird, ist eine höchst umkämpfte Angelegenheit. Gerade Großprojekte wie der Bau des Staudamms Castanhão, für dessen Fertigstellung ca. 15000 Menschen umgesiedelt wurden (Araújo, 2006:22) oder die Realisierung des Ableitungsprojektes des Rio São Francisco waren heftig umstrittene Projekte, gegen die sich starker gesellschaftlicher Widerstand regte. Mit der Fertig- 
stellung solcher Megaprojekte sind diese Konflikte und Widerstände jedoch nicht länger sichtbar. Gerade durch ihre materielle Existenz erhalten sie ihre Stabilität und ihr überindividuelles Beharrungsvermögen. Sie werden zu einem Teil von Natur und folglich zu vermeintlich unhinterfragbaren Gegebenheiten und lassen sich nur unter erheblichem Aufwand wieder verändern (Bauriedl, 2008:308).

Gleichzeitig verwandelt sich durch die Infrastruktureinrichtungen die Wasserverfügbarkeit einzelner Orte. Während in manchen Regionen mehr Wasser zugeführt wird, sinkt an anderen Orten die Verfügbarkeit sowohl des Oberflächenals auch des Grundwassers. Der soziale und politische Ursprung des materiellen Vorhandenseins bzw. Nichtvorhandenseins von Wasser - und somit von Dürreverhältnissen ist jedoch nicht sichtbar. Über materielle Objektivierungen werden soziale Unterschiede und Machtverhältnisse in natürliche Unterschiede transformiert. Eine solche Naturalisierung gesellschaftlicher Verhältnisse führt zu einer Normalisierung von Ungleichheiten und letztendlich zu einer Verfestigung von Diskursordnungen und Kräfteverhältnissen: „Die räumliche Objektivierung sozialer Tatbestände verfestigt also nicht nur bestehende soziale Ungleichheiten, womit sie sich als Hemmschuh für sozialen Wandel erweist, sie trägt zusätzlich noch zur Verschleierung sozial hergestellter Realitäten bei, indem diese der ,Natur der Dinge “ zugeschrieben werden“" (Schroer, 2008:145).

\subsubsection{Diskursivität materieller Ordnung}

Die kilometerlangen, betonierten Schneisen, die die großen Bewässerungskanäle in die Landschaft einziehen, und die riesigen Mauern großer Staudämme können geradezu als idealtypische Beispiele der Symbolkraft von Materialität angesehen werden. In ihnen kommen Vorstellungen von Fortschritt, Modernität, Staatlichkeit und der Beherrschung von Natur zum Ausdruck. Durch ihre materielle Existenz und Wirkmacht werden solche Vorstellungen (scheinbar) bestätigt. Wenn Jawaharal Nehru als Ministerpräsident Indiens Staudämme als die „Tempel der Moderne“ bezeichnet (Linton, 2008:640), dann werden diese zu einem konkreten Ort der (religiösen) Verehrung wirtschaftlicher Entwicklung und wissenschaftlichen Fortschrittes. Als ingenieurstechnische Meisterleistungen wurden Staudämme zu den wichtigsten Projekten des prometheischen Traumes der Eroberung und Kontrolle von Natur erhoben (Kaika, 2006:276). Gleichzeitig wurden sie zu zentralen Elementen der Formierung von modernen, industrialisierten Nationalstaaten, indem sie den Nationalstolz bedienten und zur Stärkung und Legitimierung von Führungspersonen und Politiken beitrugen (Molle, 2008:217; Alier, 2007:178). Genau darin ist auch der Grund zu suchen, warum der Stausee Castanhão sowohl von Präsident Fernando Henrique Cardoso, als auch von seinem Nachfolger Inácio Lula da Silva gleich zwei Mal feierlich eingeweiht wurde. Die Symbolkraft der Staumauer machte diese zu dem geeigneten Ort, um sich als einen Präsidenten zu in- szenieren, der in der Lage ist, die Dürre im Nordosten zu überwinden.

Darüber hinaus kommt der gebauten Umwelt eine ,starke Verbindlichkeit für die Wahrnehmung und das Handeln“ (Klöppel, 2010:256) zu. Über ihre spezifische Materialität werden bestimmte Denk-, Verhaltens- und Produktionsweisen hervorgebracht, andere wiederum verdrängt. So sind beispielsweise die Kanäle des Eixão das Águas in Ceará derart gestaltet, dass ein seitlicher Zugang zum Wasser nur sehr schwer möglich ist (s. Abb. 3). Eine Nutzung des Wassers durch die Kleinbäuer*innen an den Ufern des Kanals, deren Wasserversorgung oftmals vollständig von den Niederschlägen abhängt und die nicht die Position von Bewässerungslandwirt*innen einnehmen, ist somit nicht vorgesehen. Vielmehr soll das Wasser an ihnen vorbei in die Zentren der Bewässerungslandwirtschaft und in die Metropolitanregion geleitet werden.

Dabei ist die Hervorbringung von Handlungsmacht nur im Zusammenspiel mit den übrigen Elementen des Dispositivs zu sehen. Die Etablierung eines IWRM stellte den institutionellen und gesetzlichen Rahmen für die Einführung einer modernisierten Bewässerungslandwirtschaft. Dadurch wurden die Positionen von Manager*innen, Ingenieur*innen und technisch geschultem Personal gestärkt und diese zu den wichtigsten Protagonisten der Wasserpolitik erhoben. Und schließlich führte der Bau von Großstrukturen der Wasserinfrastruktur zur räumlichen Konzentration großer Wassermengen und somit zu einer Erhöhung der Wasserverfügbarkeit für die Unternehmen des Agrobusiness in der Region. Demzufolge bringt das Dürredispositiv gesellschaftliche Naturverhältnisse hervor, die sich in bestimmten Produktionsweisen, Arbeitsverhältnissen, Sozialstrukturen usw. äußern. Gleichzeitig werden über das Dispositiv andere Formen gesellschaftlicher Naturverhältnisse, wie etwa eine kleinparzellierte, dezentrale Familienlandwirtschaft sowie Vorstellungen und Praktiken des Modells der convivência com o semi-árido (Leben im Einklang mit den semiariden Verhältnissen) tendenziell zum Verschwinden gebracht.

\subsubsection{Widerstände und Brüche}

Ein solches Verständnis einer Hervorbringung von Handlungsweisen über das Dürredispositiv darf jedoch nicht als deterministische Vorgabe von Handlungsmöglichkeiten missverstanden werden. Handlungsmöglichkeiten bleiben kontingent, wenn auch nicht beliebig. Über eine Aneignung und Umdeutung der Wasserinfrastruktureinrichtungen besteht immer die Möglichkeit, Nutzungsweisen zu verändern, Widerstand zu leisten und Machtverhältnisse in Frage zu stellen. Trotz der architektonischen Gestaltung der Kanäle, der Installation von Überwachungskameras und einer institutionalisierten Nutzungsanweisung in Form des gesetzlichen Verbotes der Wasserentnahme eignen sich die Bewohner*innen an den Ufern des Kanals Eixão das Águas das Wasser an. Dies ist jedoch nicht nur als materiel- 


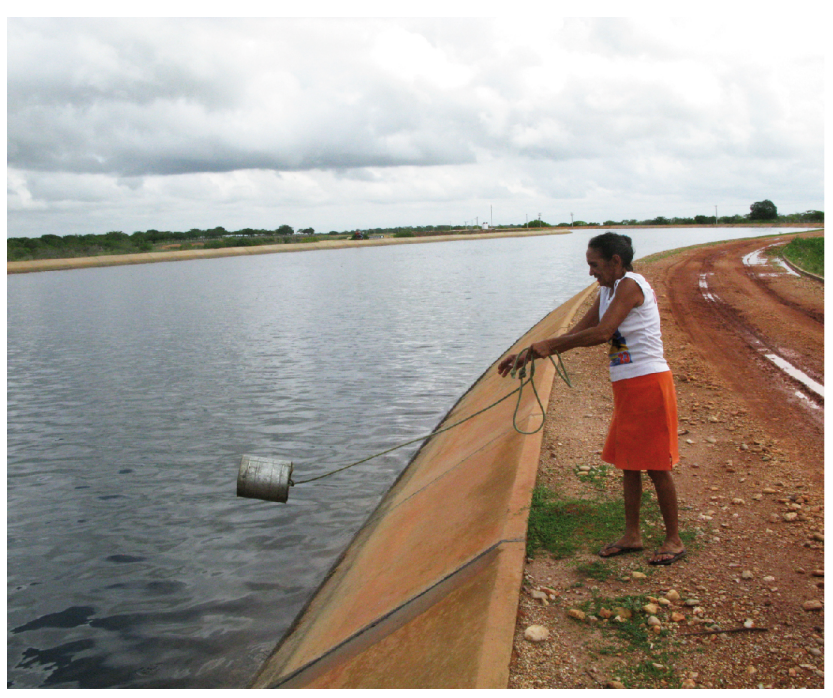

Abb. 3. Nichtvorgesehene Praktiken der Wasseraneignung.

le sondern auch als symbolische Aneignung von Wasser zu verstehen. Mit dem Bild der Wasserentnahme mit Hilfe eines einfachen Eimers aus einem randvoll gefüllten Kanal (Abb. 3) wird der hegemoniale Diskurs, dass der Wassermangel das Hauptproblem des Nordostens darstellt, herausgefordert. Über den Bruch mit den vorbestimmten Nutzungsweisen rücken Fragen nach dem Zugang zu und der Verteilung von Wasser in den Vordergrund.

Mit der Besetzung eines der wichtigsten Bewässerungskanäle auf der Hochebene von Apodi (Ceará) durch die Landlosenbewegung MST im Mai 2014 wurde die Wasserinfrastruktur von einem Instrument der einseitigen Zuteilung von Wasser und einem Medium der Reproduktion von Machtverhältnissen in ein machtvolles Instrument des Widerstandes verwandelt (Abb. 4). Die strategische Bedeutung des Kanals für die Bananenproduktion eines der größten $\mathrm{Ba}-$ nanenproduzenten Brasiliens (Banesa) erhöhte die Verhandlungsposition der Vertreter*innen des MST derart, dass der Staat in relativ kurzer Zeit bereit war, die Notwendigkeit der Landbesetzung anzuerkennen und 1700 Hektar für die Landlosenbewegung zu demarkieren. Über die diskursive Umdeutung des Kanals wurde dieser zu einem wichtigen Bestandteil bei der Verschiebung von Machtverhältnissen. Durch ihn wurden Praktiken der Aneignung ermöglicht und über ihn konstituierten sich machtvolle Subjekte des Widerstandes.

Darüber hinaus fingen die Bewohner*innen der Landbesetzung an, Felder anzulegen und das Wasser des Kanals für eine ökologisch diversifizierte Landwirtschaft zu nutzen. Schon nach einigen Monaten konnten sie die überschüssige Produktion auf den Märkten der Region verkaufen. Somit wurde über eine Umdeutung der Wasserinfrastruktur und eine veränderte Praxis der Wassernutzung die hegemoniale Logik des monokulturellen Anbaus für den Export aufgebrochen. Mit den Produkten der ökologischen Landwirtschaft materialisierte sich der Diskurs eines alternativen Ent-

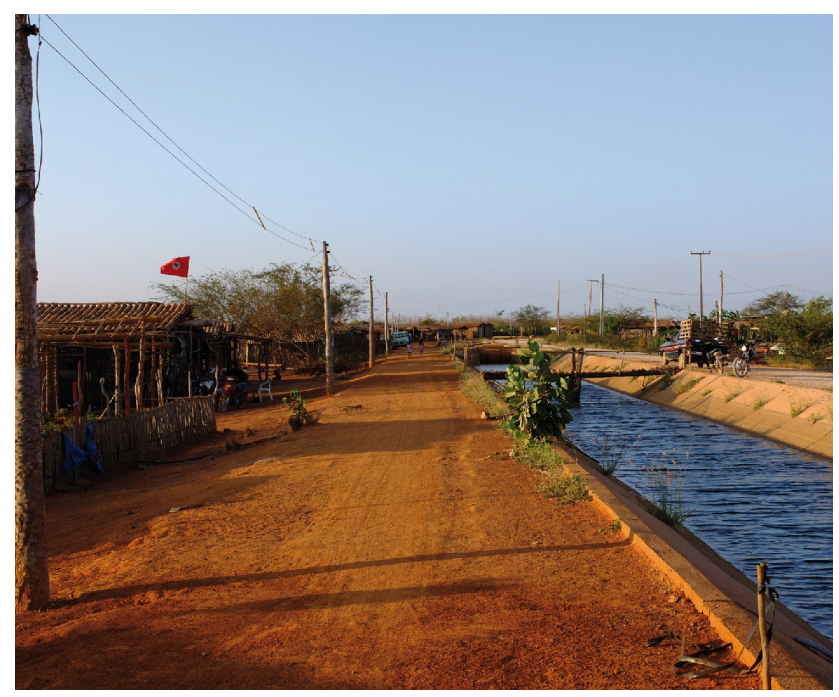

Abb. 4. Bewässerungskanal als Ausgangspunkt von Widerstand.

wicklungsmodells. In diesen Praktiken zeigt sich gleichzeitig auch das Potential, die Wasserinfrastruktur - wenn auch zunächst nur räumlich begrenzt - in ein Medium des Aufbrechens von Machtverhältnissen zu verwandeln.

\subsection{Eigenständigkeit von Materialität}

Darüber hinaus spielt in dem gegenseitigen Wechselverhältnis zwischen der diskursiven Produktion von Dürre und ihrer materiellen Einschreibung auf der einen und der Machtwirkung von Materie auf Diskurse und Handlungsweisen auf der anderen Seite auch die Eigenständigkeit von Natur eine wichtige Rolle. Natur kann nicht beliebig diskursiv geformt und angeeignet werden. Ihre spezifischen Eigenschaften sind Grundbedingungen ihrer Wahrnehmung und Verwendungsweise, auch wenn sie nie vollständig erfasst werden können. Dadurch weisen sie Widerständigkeiten gegenüber einer kompletten Integration in die Diskursordnung auf und widersetzen sich einer totalen sozialen Aneignung und Kontrolle. Diese Widerstände schränken die Kontingenz der Wirklichkeitsproduktion ein, ohne sie jedoch in lineare Kausalitätsbeziehungen zu zwängen. Wasser kann nicht beliebig transportiert, gespeichert, gemischt oder in der Bewässerungslandwirtschaft eingesetzt werden. So ist für die Umleitung des Wassers des Rio São Francisco in die Bewässerungsgebiete von Ceará ein erheblicher Energieaufwand nötig, um über $300 \mathrm{~m}$ Höhenunterschied zu überwinden. Gleichzeitig kommt es in den Staubecken und Kanälen zu erheblichen Verdunstungsverlusten und zur Verschlechterung der Wasserqualität, insbesondere durch den massiven Eintrag von Agrarchemikalien. Der intensive Einsatz von Pestiziden und Fungiziden ist jedoch nötig, um die monokulturelle Anbauweise überhaupt zu ermöglichen. Der Verfall von Bewässerungskanälen durch Nicht-Benutzung, das Brechen von Staumauern und der Ausfall von Wasserpumpen führen genauso 
zu einem Scheitern von Bewässerungsprojekten, wie der Befall durch Schädlinge und Pilze, oder das Versalzen von Böden. Auch haben die vergangenen niederschlagsarmen Jahre im Nordosten Brasiliens die große Erzählung der Inwertsetzung des Nordostens als „Kalifornien Brasiliens“ (Untied, 2005:20) erneut in Frage gestellt. Wenn der Stausee Castanhão nur noch $8 \%$ seiner Wasserspeicherkapazität aufweist, wenn die darin angelegten Vorzeigeprojekte der Fischzucht aufgrund der Wasserknappheit allesamt eingestellt werden müssen (Diário do Nordeste, 2016), dann kann der Staudamm nicht länger als Tempel der Moderne verehrt werden sondern wird eher als Wahrzeichen des gescheiterten Versuchs der Naturbeherrschung gelesen. Die spezifische Materialität von Natur ist letztlich eine Quelle von ,unpredictability, unruliness and, in some cases, resistance to human intentions" (Bakker und Bridge, 2006:18). Dabei kann ein Scheitern der hegemonialen Aneignungsstrategien aufgrund der Widerständigkeit von Natur und Materialität neue diskursive Ereignisse hervorbringen, die wiederum zu Verschiebungen innerhalb der Diskursordnung und letztlich auch innerhalb des Dispositivs führen.

\section{Fazit}

Am Beispiel der Expansion der Bewässerungslandwirtschaft im Nordosten Brasiliens wird deutlich, dass eine Analyse von Diskursen allein nicht ausreichend erscheint, um die in den letzten Jahrzehnten hegemonial gewordene, spezifische Inwertsetzung von Natur angemessen zu erklären. Der monokulturelle Anbau für den Export im Kontext einer semiariden Region kann nicht nur mit Diskursen über Wassermangel und Ressourceneffizienz begründet werden. Vielmehr müssen die unterschiedlichen Ebenen und Dimensionen einer gesellschaftlichen Situation erörtert und in einen Gesamtzusammenhang eingebettet werden. Mit dem Konzept der Dispositivanalyse, das den Fokus der Untersuchungen explizit auf die Verbindungen zwischen den unterschiedlichen Dimensionen gesellschaftlicher Naturverhältnisse legt, können die Vermittlungszusammenhänge zwischen gesellschaftlichen Verhältnissen und materiellen Bedingungen herausgearbeitet werden, ohne auf essentialisierende und dichotome Beschreibungen zurückgreifen zu müssen. Wie Dürren diskursiv gerahmt und bspw. über Besitzverhältnisse und Zugangsrechte zu Wasser gesellschaftlich vermittelt werden, ist dabei ebenso Gegenstand der Untersuchungen, wie die Frage danach, wie über materielle Bedingungen Machtverhältnisse (re)produziert werden.

Durch die Anerkennung der Eigenständigkeit der einzelnen Elemente des Dispositivs, bei gleichzeitigem Insistieren auf ihre unausweichliche Verbundenheit, können Diskurse, Institutionalisierungen, Subjektpositionen, Praktiken und Materialität sowohl in ihrer Eigenlogik untersucht als auch nach ihren gegenseitigen Wechselbeziehungen befragt werden. Erst durch die Berücksichtigung der institutionel- len Verankerung der Diskurse über Gesetze, Organisationsformen und Finanzierungsmechanismen werden der grundlegende Charakter der Veränderungen und ihr potentielles Beharrungsvermögen offenbar.

Am Beispiel der Analyse der Wasserinfrastruktur in der Region Baixo Jaguaribe konnte gezeigt werden, wie sich Diskurse entlang von Machtstrukturen in die Umwelt einschreiben und manifest werden. Durch den spezifischen materiellen Charakter der Kanäle und Staudämme werden Wahrnehmungs- und Handlungsweisen vorstrukturiert und Diskursordnungen auf Dauer gestellt. Somit ist die Materialität der Wasserinfrastruktur nicht nur Ergebnis von Diskursen und sozialen Praktiken, sie stellt auch deren Basis und Ausgangsbedingung dar. Dabei gilt es, sich nicht in einer „Henne-oder-Ei“"-Diskussion zu verlieren, sondern auf die permanente Gleichzeitigkeit des Vermittlungsverhältnisses zu verweisen.

Durch die Fokussierung auf die Wechselbeziehungen zwischen den unterschiedlichen Elementen kann die gegenseitige Herstellung diskursiver und materieller Elemente und die Agency von Materialität herausgearbeitet werden. Über die gebaute Umwelt in Form von Staumauern, Schleusen und Kanälen kann Wasser auf eine spezifische Art und Weise gespeichert, transportiert und verteilt werden. Nur dadurch wird eine bestimmte Zuteilung, Nutzung und Kontrolle von Wasser überhaupt möglich. Dabei geht es jedoch nicht darum, die Stauseen und Kanäle als eine Art neue, intentional handelnde Akteure zu bestimmen, die den Wasserzugang selbstbestimmt regeln. Vielmehr werden erst im Zusammenspiel zwischen Diskursen, Institutionalisierungen, Subjektpositionen, Praktiken und Materialität bestimmte Aneignungsweisen von Natur hervorgebracht und hegemonial.

Der Verweis auf die Eigenlogik der einzelnen Elemente innerhalb des Dispositivs bedeutet gleichzeitig auch, die Eigenständigkeit von Materialität ernst zu nehmen und das Verständnis von Materie als passives Rohmaterial zu überwinden. Materialität kann demnach nicht beliebig diskursiv hergestellt werden und entzieht sich somit gleichzeitig einer völligen Beherrschbarkeit. Die Nicht-Identität von Natur ist insbesondere dann bestimmbar, wenn diskursive Einschreibungen, institutionelle Vorstrukturierungen oder vorgesehene Aneignungsweisen scheitern oder zu unvorhergesehen Ergebnissen führen. Somit kann es auch aufgrund der Eigenlogik der Materialität zu Veränderungen des Dispositivs kommen. Dadurch verschieben sich bestehende Konstellationen und hegemoniale Verfestigungen können aufgebrochen werden. Zusammenfassend stellt das Konzept einer an Foucault angelehnten Dispositivanalyse eine Möglichkeit dar, die Wechselverhältnisse zwischen Diskursivität und Materialität analytisch zu rahmen. Gerade da innerhalb einer Dispositivanalyse die Beziehungen zwischen sozialen Verhältnissen und materiellen Gegebenheiten explizit auf ihre Machtwirkungen hin befragt werden, bietet das Konzept die Möglichkeit, „die materiellen Facetten eines poststrukturalistischen Machtkonzepts hervorzuheben und den Zwischen- 
raum symbolischer und materieller Ordnungen zum Schauplatz geographischer Forschung zu machen" (Mattissek und Wiertz, 2014:158). Darüber hinaus stellt ein solcher Ansatz insbesondere für empirisch angelegte Arbeiten innerhalb der Geographie die Möglichkeit dar, diskursanalytische Untersuchungen mit ethnographischen Methoden konzeptionell zu verbinden, um dadurch die Komplexität gesellschaftlicher Naturverhältnisse besser in den Blick nehmen zu können.

\section{Datenverfügbarkeit}

In dem Artikel beziehe ich mich vor allem auf Interviews und Erhebungen, die ich im Rahmen meiner Dissertation durchgeführt habe und die nicht öffentlich zugänglich sind. Eine ausführliche Besprechung und Auswertung der Daten findet sich in Schmitt (2013).

Danksagung. Bedanken möchte ich mich vor allem bei Sören Becker für die vielen Anmerkungen und produktiven Kommentare und bei den beiden unbekannten Gutachter*innen, die wesentlich zur Schärfung der Argumente und zum Entstehen des Artikels in seiner jetzigen Form beigetragen haben.

Edited by: S. Becker

Reviewed by: two anonymous referees

\section{Literatur}

ADECE (Agência do Desenvolvimento do Estado do Ceará): Relação de empresas com reserva de área CIPP, Base CEDE/ADECE 2011-2013, o. O., 2013.

ADECE (Agência de Desenvolvimento do Estado do Ceará): Eixão das Águas, verfügbar unter: http://www.adece.ce.gov. br/index.php/recursos-hidricos/eixao-das-aguas (letzter Zugriff: 24.02.2016), 2015.

Adorno, T. W.: Spengler nach dem Untergang, Zu Oswalds Spenglers 70. Geburtstag, Der Monat, 115-127, 1950.

Adorno, T. W.: Soziologische Schriften I, Suhrkamp, Frankfurt am Main, 587 pp., ISBN: 978-3-518-29308-9, 2003.

Albuquerque, D. M. d.: A invenção do nordeste e outras artes, Cortez Editora, Recife, São Paulo, 376 pp., ISBN: 9788524914850 , 1999.

Alier, J. M.: O ecologismo dos pobres, Editora Contexto, São Paulo, 379 pp., ISBN: 9788572443586, 2007.

Araújo, C. E. de: Movimentos dos Atingidos por Barragens (MAB), A questão ambiental e a participação política, Dissertação de Mestrado, UFC (Universidade Federal do Ceará), Fortaleza, 145 pp., 2006.

Bakker, K.: From state to market?: water mercantilización in Spain, Environment and Planning A, 34, 767-790, 2002.

Bakker, K. J.: An uncooperative commodity, Privatizing water in England and Wales, Oxford University Press, Oxford, New York, 224 pp., ISBN: 9780199253654, 2003.

Bakker, K. und Bridge, G.: Material worlds? Resource geographies and the 'matter of nature', Prog. Hum. Geog., 30, 5-27, 2006.
Bartelt, D. D.: Kosten der Modernisierung. Der Sertão des brasilianischen Nordostens in der zweiten Hälfte des 19. Jahrhunderts: zwischen Homogenisierung und Diskurs, Jahrbuch für Geschichte Lateinamerikas, 38, 327-351, 2001.

Bauriedl, S.: Räume lesen lernen: Methoden zur Raumanalyse in der Diskursforschung, Hist. Soc. Res., 33, 278-312, 2008.

Becker, E., Hummel, D., und Jahn, T.: Gesellschaftliche Naturverhältnisse als Rahmenkonzept, in: Matthias Groß (Hrsg.): Handbuch Umweltsoziologie, VS Verl. für Sozialwiss, Wiesbaden, 75-96, 2011.

Blaikie, P. M. und Brookfield, H. C.: Land degradation and society, Methuen, London, New York, 299 pp., ISBN 0-416-40140-6, 1987.

Bouleau, G.: The co-production of science and waterscapes: The case of the Seine and the Rhône Rivers, France, Geoforum, 57, 248-257, 2014.

Bryant, R. L. und Bailey, S.: Third World political ecology, Routledge, London, New York, 237 pp., ISBN 9780415127448, 1997.

Budds, J.: Power, Nature and Neoliberalism:, The Political Ecology of Water in Chile, Singapore J. Trop. Geo., 25, 322-342, 2004.

Budds, J.: Whose Scarcity? The Hydrosocial Cycle and the Changing Waterscape of La Ligua River Basin, Chile, in: Contentious geographies: Environmental knowledge, meaning, scale, Herausgeber: Goodman, M. K., Boykoff, M. T., und Evered, K. T., Ashgate, Hampshire, 59-78, 2008.

Bührmann, A. und Schneider, W.: Vom Diskurs zum Dispositiv, Eine Einführung in die Dispositivanalyse, transcript, Bielefeld, 180 pp. ISBN: 978-3-89942-818-6, 2008.

Castree, N.: Marxism and the Production of Nature, Capital \& Class, 5-36, 2000.

Castree, N.: Socializing Nature: Theory, Practice, and Politics, in: Social nature, Theory, practice, and politics, Herausgeber: Castree, N. und Braun, B., Blackwell Publishers, Malden, Mass, 1-21, 2001.

de Carvalho, O.: A economía política do Nordeste, Secas, irrigação e desenvolvimento, Editora Campus, Rio de Janeiro, 505 pp., ISBN: 9788570014986, 1988.

Diário do Nordeste: Produção da tilápia tem queda de quase $100 \%$ no Castanhão, http:// diariodonordeste.verdesmares.com.br/cadernos/regional/ producao-da-tilapia-tem-queda-de-quase-100-no-castanhao- 1 . 1582145, 13 Juli 2016.

Elias, D. und Sampaio, J. L. F. (Hrsg.): Modernização Excludente, Paradigmas da Agricultura Cearense, Fortaleza, Verlag: Edições Demócrito Rocha, 160 pp., ISBN: 8575290657, 2002.

Escobar, A.: Postconstructivist political ecologies, in: The international handbook of environmental sociology, Redclift, M. R. und Woodgate, G., Edward Elgar, Cheltenham, Northampton, 91105, 2010.

Foucault, M.: Archäologie des Wissens, Suhrkamp, Frankfurt am Main, 312 pp., ISBN: 978-3-518-27956-4, 1973.

Foucault, M.: Überwachen und Strafen, Die Geburt des Gefängnisses, 1. Aufl., Suhrkamp, Frankfurt am Main, 408 pp., ISBN: $978-$ 3-518-38771-9, 1977.

Foucault, M.: Dispositive der Macht, Über Sexualität, Wissen und Wahrheit, Merve, Berlin, 232 pp., ISBN-10: 3920986962, 1978.

Foucault, M.: Der Wille zum Wissen, Suhrkamp, Frankfurt am Main, 153 pp., ISBN: 978-3-518-28316-5, 1983. 
Gandy, M.: Rethinking urban metabolism: water, space and the modern city, City, 8, 363-379, 2004.

Görg, C.: Nichtidentität und Kritik. Zum Problem der Gestaltung der Naturverhältnisse, in: Kritische Theorie der Technik und der Natur, Herausgeber: Böhme, G. und Manzei, A., Fink, München, 113-133, 2003a.

Görg, C.: Regulation der Naturverhältnisse, $\mathrm{Zu}$ einer kritischen Theorie der ökologischen Krise, Westfälisches Dampfboot, Münster, 345 pp., ISBN: 3-89691-530-4, 2003b.

Görg, C.: Raum und Gesellschaft, Zur Bedeutung von Ort und Raum in Zeiten der "Globalisierung", in: Strukturierung von Raum und Landschaft, Konzepte in Ökologie und der Theorie gesellschaftlicher Naturverhältnisse, Herausgeber: Weingarten, M., 1. Aufl., Westf. Dampfboot, Münster, 222-239, 2005.

Haraway, D.: Simians, cyborgs and women : the reinvention of nature, Routledge, New York, 312 pp., ISBN-10: 0415903874, 1991.

Harvey, D.: Justice, nature and the geography of difference, 11. Aufl., Blackwell, Cambridge, 480 pp., ISBN-10: 1557866813, 2007.

Horkheimer, M.: Kritik der instrumentellen Vernunft, Fischer, Frankfurt am Main, 205 pp., ISBN: 9783596178209, 1967.

ICWE (International Conference on Water and Environment): The Dublin Statement and Report of the Conference, International Conference on Water and the Environment, Dublin, 1992.

IPECE (Instituto de Pesquisa e Estratégia Econômica do Ceará): Índice Municipal de Alerta (IMA) 2007, Um Instrumento para Orientações Preventivas sobre as Adversidades Climáticas Estado do Ceará - 2007, Fortaleza, 2007a.

IPECE (Instituto de Pesquisa e Estratégia Econômica do Ceará): Ceará em Mapas, verfügbar unter: http://www2.ipece.ce.gov.br/ atlas/capitulo5/51.htm (letzter Zugriff: 28.02.2013), 2007b.

Jäger, S.: Dispositiv, in: Michel Foucault, Eine Einführung in sein Denken, Herausgeber: Kleiner, S., Campus, Frankfurt am Main, 72-89, 2001.

Jahn, T. und Wehling, P.: Gesellschaftliche Naturverhältnisse Konturen eines theoretischen Konzepts, in: Soziologie und Natur, Theoretische Perspektiven, Herausgeber: Brand, K.-W., Leske + Budrich, Opladen, 75-93, 1998.

Kaika, M.: City of flows: modernity, nature, and the city, Routledge, New York, Abingdon, 216 pp., ISBN-10: 0415947162, 2005.

Kaika, M.: Dams as Symbols of Modernization: The Urbanization of Nature Between Geographical Imagination and Materiality, Ann. Assoc. Am. Geogr., 96, 276-301, 2006.

Kaika, M.: City of Flows. Der Wandel der symbolischen Bedeutung technischer Infrastrukturen in der Moderne, in: Infrastrukturnetze und Raumentwicklung, Zwischen Universalisierung und Differenzierung, Herausgeber: Moss, T., Naumann, M., und Wissen, M., Oekom-Verl., München, 87-111, 2008.

Kazig, R. und Weichhart, P.: Die Neuthematisierung der materiellen Welt in der Humangeographie, Berichte zur deutschen Landeskunde, 83, 109-128, 2009.

Keller, R.: Diskursforschung, Eine Einführung für SozialwissenschaftlerInnen, VS Verlag für Sozialwissenschaften, Wiesbaden, 136 pp., ISBN 978-3-531-17352-8, 2007.

Keller, R.: Wissenssoziologische Diskursanalyse, Grundlegung eines Forschungsprogramms, VS Verlag für Sozialwissenschaften, Wiesbaden, 360 pp., ISBN: 978-3-531-17837-0, 2008.
Klöppel, U.: Foucaults Konzept der Problematisierungsweise und die Analyse diskursiver Transformationen, in: Diskursiver Wandel, Herausgeber: Landwehr, A., VS, Verl. für Sozialwiss., Wiesbaden, 255-263, 2010.

Köhler, B.: Gesellschaftliche Naturverhältnisse, Politische Ökologie und ökologisch-feministische Sichtweisen, Das Argument, 50, 850-857, 2008.

Köhler, B. und Wissen, M.: Gesellschaftliche Naturverhältnisse, Ein kritischer theoretischer Zugang zur ökologischen Krise, in: Kritische politische Bildung, Ein Handbuch, Herausgeber: Lösch, B. und Andreas Thimmel, A., Wochenschau-Verlag, Schwalbach, 217-227, 2010.

Latour, B.: Wir sind nie modern gewesen, Versuch einer symmetrischen Anthropologie, Ungek. Ausg., Fischer-Taschenbuch-Verl, Frankfurt am Main, 208 pp., ISBN: 9783596137770, 1998.

Linton, J.: Is the Hydrologic Cycle Sustainable?, A HistoricalGeographical Critique of a Modern Concept, Ann. Assoc. Am. Geogr., 98, 630-649, 2008.

Linton, J.: What is water?, The history of a modern abstraction, UBC Press, Vancouver, 352 pp., ISBN: 978-0-7748-1701-1, 2010.

Linton, J. und Budds, J.: The hydrosocial cycle: Defining and mobilizing a relational-dialectical approach to water, Geoforum, 57, 170-180, 2014.

Lykke, N.: Feministischer Postkonstruktionismus, in: Critical Matter, Herausgeber: in: Goll, T., Keil, D., und Telios, T., 36-48, 2013.

Marx, K.: Das Kapital, Band I, Dietz, Berlin, 955 pp., 1968.

Mattissek, A. and Wiertz, T.: Materialität und Macht im Spiegel der Assemblage-Theorie: Erkundungen am Beispiel der Waldpolitik in Thailand, Geogr. Helv., 69, 157-169, doi:10.5194/gh-69-1572014, 2014.

Molle, F.: Why Enough Is Never Enough: The Societal Determinants of River Basin Closure, Water Resources Development, 24, 217-226, 2008.

Monstadt, J.: Conceptualizing the political ecology of urban infrastructures: insights from technology and urban studies, Environ. Plann. A, 41, 1924-1942, 2009.

Murdoch, J.: Inhuman/nonhuman/human: actor-network theory and the prospects for a nondualistic and symmetrical perspective on nature and society, Environ. Plann. D, 15, 731-756, 1997.

Namberger, V.: Rassismustheorien und die Materialität des Körpers, in: Critical Matter, Herausgeber: Goll, T., Keil, D., und Telios, T., 134-150, 2013.

Pels, D., Hetherington, K., und Vandenberghe, F.: The Status of the Object, Performances, Mediations, and Techniques, Theory, Culture \& Society, 19, 1-21, 2002.

Robbins, P.: Political ecology, A critical introduction, Blackwell Pub., Malden, MA, 242 pp., ISBN-10: 1405102667, 2004.

Schmitt, T.: Dürre als gesellschaftliches Naturverhältnis, Eine politische Ökologie der Wasserknappheit im Nordosten Brasiliens, Dissertation, Universität Innsbruck, Innsbruck, Institut für Geographie, 425 pp., 2013.

Schmitt, T.: Integrated Water Resources Management in Brazil: Participatory Approaches as a Way to Resource Justice?, in: Fairness and Justice in Natural Resource Politics, Herausgeber: Pichler, M., Staritz, C., Küblböck, K., Plank, C., Raza, W., und Ruiz Peyré, F., Routledge, London, New York, 73-89, 2016. 
Schroer, M.: Raum, Das Ordnen der Dinge, in: Poststrukturalistische Sozialwissenschaften, Herausgeber: Moebius, S. und Reckwitz, A., Suhrkamp, Frankfurt am Main, 141-157, 2008.

Smith, N.: Uneven Development: Nature, Capital and the Production of Space, Blackwell, Oxford, 198 pp., ISBN 10: 0631136851, 1984.

SRH (Secretaria dos Recursos Hídricos): Consolidação da Política do Programas de Recursos Hídricos do Estado do Ceará, Atualização do Plano Estadal de Recursos Hídricos, Fortaleza, 2005.

Swyngedouw, E.: Modernity and Hybridity: Nature, Regeneracionismo, and the Production of the Spanish Waterscape, 18901930, Ann. Assoc. Am. Geogr., 89, 443-465, 1999.

Swyngedouw, E.: Liquid power, Contested hydro-modernities in twentieth-century Spain, MIT Press, Cambridge, Massachusetts, 320 pp., ISBN: 9780262029032, 2015.
Taddei, R.: Of Clouds and Streams, Prophets and Profits: The political semiotics of climate and water in the brazilian Northeast, Dissertation, Columbia University, New York, verfügbar unter: http://citeseerx.ist.psu.edu/viewdoc/download;jsessionid= 3BEC24EEA353E5E3402175A7A9C2D25C?doi=10.1.1.85. 4893\&rep=rep1\&type=pdf (letzter Zugriff: 15.06.2010), 2005.

Untied, B.: Bewässerungslandwirtschaft als Strategie zur kleinbäuerlichen Existenzsicherung in Nordost-Brasilien?, Handlungsspielräume von Kleinbauern am Mittellauf des São Francisco, Dissertation, Universität Marburg, Marburg, Institut für Geographie, verfügbar unter: http://archiv.ub.uni-marburg.de/diss/ z2005/0531/pdf/dbu.pdf (letzter Zugriff: 02.09.2010), 2005.

Wissen, M.: Gesellschaftliche Naturverhältnisse in der Internationalisierung des Staates, Konflikte um die Räumlichkeit staatlicher Politik und die Kontrolle natürlicher Ressourcen, Westfälisches Dampfboot, Münster, 301 pp., ISBN: 9783896918789, 2011. 